\author{
SERIES "RECENT DEVELOPMENTS IN PULMONARY INFECTIONS" \\ Edited by M. Woodhead and T. Schaberg \\ Number 8 in this Series
}

\title{
Influenza: vaccination and treatment
}

\author{
I. Stephenson, K.G. Nicholson
}

\begin{abstract}
Influenza: vaccination and treatment. I. Stephenson, K.G. Nicholson. CERS Journals Ltd 2001.

ABSTRACT: Few conditions exert such an enormous toll of absenteeism, suffering, medical consultations, hospitalization, death and economic loss as influenza. Patients at high risk of complications and mortality include the elderly and those with pre-existing cardiopulmonary disease.

The outbreak in 1997 in Hong Kong, of avian H5N1 influenza in man, which resulted in six deaths among 18 hospitalized cases, and the recent isolation of H9N2 viruses from two children in Hong Kong, are reminders that preparation must be made for the next pandemic. Since the 1970 s, efforts to control influenza have mostly focussed on the split product and surface antigen vaccines. These vaccines are of proven efficacy in healthy adults and are effective in elderly people with and without medical conditions putting them at high risk of complications and death following influenza infection.

However, vaccine coverage is patchy and often low, and outbreaks of influenza are not uncommon in well-immunized residents of nursing homes. New vaccines and methods of vaccine delivery are being developed in attempts to overcome the limitations of existing vaccines.

The antiviral drugs amantadine and rimantadine were developed in the 1960s, but have not been used widely due to their spectrum of activity, rapid emergence of resistance, and adverse effects associated with amantadine. The site of enzyme activity of the influenza neuraminidase is highly conserved between types, subtypes and strains of influenza and has emerged as the target of an exciting new class of antiviral agents that are effective both prophylactically and as therapy.
\end{abstract}

Eur Respir J 2001; 17: 1282-1293.

Dept of Infection and Tropical Medicine, Leicester Royal Infirmary, Leicester, UK.

Correspondence: I. Stephenson, Dept of Infection and Tropical Medicine, Leicester Royal Infirmary, Leicester LE1 5WW, UK.

Fax: 441162585067

Keywords: Antiviral drugs influenza neuraminidase

vaccination

Received: September 252000

Accepted after revision October 32000
Influenza is a contagious respiratory viral illness of global importance. Influenza viruses are unique among respiratory viruses with their segmented genome and great antigenic diversity. They have been classified into three distinct types: A, B and C. Influenza $\mathrm{A}$ is responsible for occasional pandemics involving millions of people worldwide and frequent, usually annual epidemics that are often associated with considerable morbidity and mortality. Outbreaks of influenza B are less frequent and are associated with a lower burden of illness overall. Influenza $\mathrm{C}$ is associated with sporadic and usually asymptomatic infection. At least three pandemics occurred during the last century. By far the worst was the pandemic of 1918-1920. Over a 10-month period, this "Spanish flu" killed an estimated 20-40 million people worldwide with many of the deaths occurring in young, previously healthy adults.

When symptomatic, influenza typically produces an acute febrile illness characterized by cough, headache and myalgia. The illness is generally debilitating, often causing several days of restricted activity including lost school or work days. The majority of morbidity and mortality occurs in the elderly with underlying chronic cardiorespiratory disease or diabetes mellitus, particularly those in residential care. Complications of acute influenza include viral and secondary bacterial pneumonias, exacerbations of pre-existing cardiopulmonary disease and, in children and infants, croup, otitis media, bronchiolitis and febrile convulsions [1, 2].

Previous articles in this series: No. 1: Hammerschlag MR. Chlamydia pneumoniae and the lung. Eur Respir J 2000; 16: 1001-1007. No. 2: Ewig S, Schäfer H, Torres A. Severity assessment in community-acquired pneumonia. Eur Respir J 2000; 16: 1193-1201. No. 3: Nicod LP, Pache J-C, Howarth N. Fungal infections in transplant recipients. Eur Respir J 2001; 17: 133-140. No. 4: Jones AM, Dodd ME, Webb AK. Burkholderia cepacia: current clinical issues, environmental controversies and ethical dilemmas. Eur Respir J 2001; 17: 295-301. No. 5: Rook GAW, Seah G, Ustianowski A. M. tuberculosis: immunology and vaccination. Eur Respir J 2001; 17: 537-557. No. 6: Ioanas M, Ferrer R, Angrill J, Ferrer M, Torres A. Microbial investigation in ventilator-associated pneumonia. Eur Respir J 2001; 17: 791-801. No. 7: Wilson R. Bacteria, antibiotics and COPD. Eur Respir J 2001; 17: 995-1007. 
During influenza outbreaks, hospital admission rates for respiratory illnesses increase, as does mortality from all causes [3].

\section{Structure and classification}

Influenza viruses are enveloped ribonucleic acid (RNA) viruses with a segmented genome belonging to the family of Orthomyxoviridae. Both influenza A and $B$ viruses contain eight RNA segments, each individually encapsidated by the viral nucleoprotein, and possess two surface glycoproteins embedded into the membrane: the haemagglutinin (HA) and neuraminidase (NA), which are capable of eliciting antibody responses in humans. Influenza $\mathrm{C}$ viruses contain seven RNA segments. Haemagglutinin is involved with receptor binding and membrane fusion. The neuraminidase enzyme catalyses the cleavage of viral progeny from infected cells. It also prevents virus clumping so that each virion can function as an independent infectious unit. It facilitates cleavage of sugar residues on the $\mathrm{HA}$, and by modifying $\mathrm{HA}$ carbohydrate side chains, may be implicated in virulence. It also facilitates movement of the virus through inhibitory mucopolysaccharides coating the respiratory tract epithelium, allowing cell to cell spread through the respiratory mucosa. A third surface protein, the M2 ion channel, is a tetrameric membrane channel important in the regulation of internal $\mathrm{pH}$ of the virion. By allowing the acidification necessary for the uncoating of viral ribonucleoprotein, the M2 ion channel plays a pivotal role in viral replication [4].

Strains of influenza are classified on the basis of their core proteins into types (i.e. A, B or C), by host species of origin (e.g. avian, porcine), geographic site of isolation, serial number, year of isolation and, for influenza A viruses, by subtypes of $\mathrm{HA}$ and NA antigens. A total of 15 HAs $(\mathrm{H} 1-\mathrm{H} 15)$ and nine NAs (N1 - N9) have been identified for influenza A within its natural avian reservoir. Only one novel HA has been discovered in the past $10 \mathrm{yrs}$, despite extensive surveillance of influenza in humans and animals, implying that the number of $\mathrm{HA}^{\prime}$ s and $\mathrm{NA}^{\prime} \mathrm{s}$ in nature is finite. Despite the presence of $15 \mathrm{HAs}$ in nature, only a few (H1, H2 and $\mathrm{H} 3$ ) have formed stable lineages in man during the last century implying host specificity. The HA of human influenza viruses binds preferentially to terminal sialic acid with an $\alpha 2,6$ linkage to galactose on cell surfaces. In contrast avian influenza viruses preferentially bind $\alpha 2,3$-galactose linkages. Human epithelial cells contain sialic acid a2,6-galactose linkages, but not sialic acid $\alpha 2,3-$ galactose linkages. In contrast, avian intestinal epithelial cells (the principal site of replication in birds) contain sialic acid $\alpha 2,3$-galactose linkages, but not $\alpha 2,6$-galactose linkages. The pig trachea contains receptors for both avian and human influenza viruses and the domestic pig supports the growth of viruses of both human and avian origin. For this reason, it has been proposed that genetic reassortment between avian and human virus, leading to a novel strain, may occur in pigs and that pigs represent a "mixing bowl" for the evolution of human pandemic strains
[5]. However, it is now recognized that avian viruses can replicate in humans.

\section{Antigenic shift and drift}

Pandemics and epidemics arise as a result of changes in the HA. Pandemic influenza is the outcome of "antigenic shift" which reflects a major change in the HA and possibly NA and occurs only with influenza A virus. Antigenic shift occurs as a result of genetic reassortment between the avian and human pools when a virus with a "new" HA emerges (i.e. a new subtype) which is serologically distinct from earlier viruses circulating in humans and could not have arisen from these viruses by mutation. There is little or no background immunity in the population to the new virus so it spreads rapidly, usually causing extensive morbidity and mortality. Interpandemic outbreaks are caused by viruses that have undergone minor antigenic changes or "drift". These strains are generated from the accumulation of random point mutations in the genome at sites coding for exposed sections of either HA or NA [6]. Both antigenic shift and drift pose problems for vaccine production.

It is inevitable that another pandemic will occur at some point in the future. During March-May 1997, an outbreak of avian H5N1 influenza in chickens in the New Territories of Hong Kong affected three chicken farms, with an overall mortality of $70 \%$ among the 6800 chickens. In May and November/ December 1997, cases of influenza due to avian H5N1 viruses occurred in humans in Hong Kong, causing six deaths (33\% mortality) among 18 hospital admissions [7]. Extensive analyses revealed that the human isolates were of avian origin and not been derived by reassortment. Clearly, had the virus spread more widely, it could have resulted in a pandemic causing many millions of deaths. Influenza H9N2 virus is widespread in poultry in Asia and has recently been recovered from two children with mild, self limiting illness [8]. These experiences highlight the need for vigilance, and surveillance of both animals and man for influenza, and effective vaccines and antiviral therapies.

\section{Inactivated vaccines}

Vaccination represents the mainstay for influenza prevention. Antibodies directed against the haemagglutinin and neuraminidase glycoproteins are associated with protection against infection and amelioration of illness. Three types of inactivated influenza vaccine are currently available in the world: whole virus, split product and purified surface antigen vaccines. All commercially produced, inactivated influenza vaccines are grown in eggs.

Early whole virion inactivated vaccines were associated with frequent local and systemic adverse effects. Although very pure, whole virion vaccines still produce febrile reactions and are unsuitable for use in young children. Accordingly, whole virion influenza vaccines are little used and are unlicensed in many 
countries. Most influenza vaccines are supplied as "split" vaccines, produced from the disrupted highlypurified influenza virus, or as "surface antigen" vaccines containing predominantly purified haemagglutinin and neuraminidase. Split and surface antigen vaccines are as immunogenic as whole virion vaccines in primed individuals, but two doses are required in young children and also during pandemics.

Influenza vaccines evoke a strain-specific response with reduced levels of protection against viruses produced by antigenic drift. Influenza vaccines are ineffective against unrelated strains. Accordingly, vaccine composition for the northern hemisphere is reviewed in February by the World Health Organization (WHO) and for the southern hemisphere in September, and the antigenic makeup updated depending on surveillance of the current prevalent circulating subtypes to provide vaccines well matched antigenically to strains that are expected to cause epidemics. In the decade 1987-1997, no changes were made in recommendations for the $\mathrm{A} / \mathrm{H} 1 \mathrm{~N} 1$ strain, nine changes were made for the $\mathrm{A} / \mathrm{H} 3 \mathrm{~N} 2$ strain and four changes for the B strain. A good match between wildtype and vaccine strains was made in respect of 23 $(77 \%)$ of 30 circulating strains, but when considered on an annual basis, the epidemic strains differed from the vaccine strains during 5 of 10 seasons.

Current inactivated vaccines are usually trivalent, containing $15 \mu \mathrm{g}$ each of two influenza A subtypes (H1N1 and H3N2) and one influenza B strain. Following vaccination with influenza $A, \sim 90 \%$ of normal subjects achieve serum haemagglutinin-inhibition (HAI) titres of $>1: 40$, a level generally associated with protection of about $50 \%$ of the population [9]. Protection against laboratory confirmed influenza $\mathrm{A}$ illness of $70-95 \%$ in young healthy subjects can be achieved when there is a good match between vaccine and circulating strains [10]. Few trials have studied the efficacy of the influenza vaccine in the elderly. A randomized, controlled trial conducted in Australia in 1969 showed that the monovalent influenza vaccine was associated with a $62 \%$ reduction in laboratoryconfirmed illness [11]. In Holland, during the 1991 - 1992 season when vaccine and epidemic strains were well matched, vaccination of elderly, mostly healthy subjects, was associated with an overall $58 \%$ reduction in clinical and laboratory-confirmed influenza [12] (fig. 1).

Because placebo-controlled trials of the influenza vaccine are considered unethical in the elderly and/or at risk populations, recent studies have focused on the effectiveness of the influenza vaccine in preventing hospitalization for "pneumonia and influenza", all respiratory conditions, and deaths. Whether conducted as cohort or case-control studies, numerous studies have demonstrated effectiveness, both during influenza $A$ and $B$ seasons. In noninstitutionalized elderly people, vaccination is associated with a $19-63 \%$ reduction in hospitalization for pneumonia and influenza, a $17-39 \%$ reduction in hospitalization for all respiratory conditions, and a $27-75 \%$ reduction in all causes of mortality [13] (fig. 2). Comparable levels of effectiveness have been demonstrated in high-, intermediate-, and low-risk individuals [17].

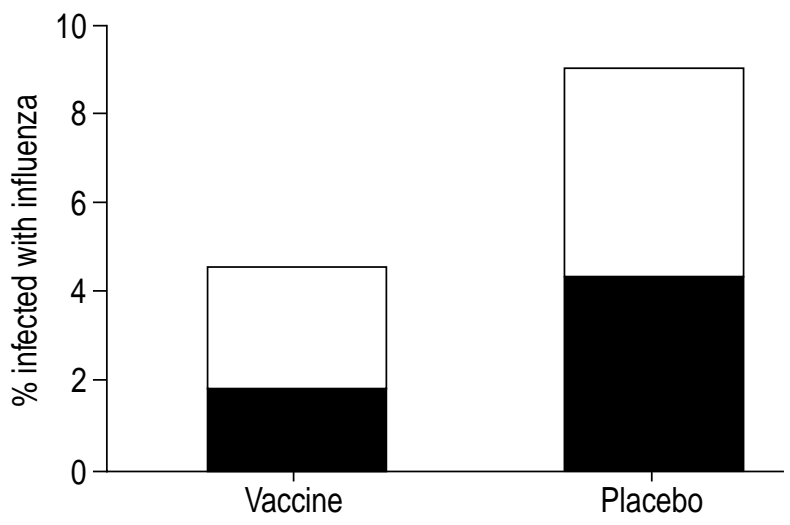

Fig. 1. - Efficacy of influenza vaccination $(n=901)$ and placebo $(\mathrm{n}=889)$ in the elderly: a randomized double-blind, placebocontrolled trial. $\square$ : subclinical influenza (relative risk $(R R)=0.59$ $(95 \%$ confidence interval $(95 \% \mathrm{CI})=0.36-0.96))$; $\mathbf{\square}$ : symptomatic influenza $(\mathrm{RR}=0.42(95 \% \mathrm{CI}=0.23-0.74))$. Adapted from [12].

These estimates of clinical effectiveness are determined by the observed reductions in clinically-relevant but nonspecific outcomes (i.e. not laboratory-confirmed), such as influenza-like illnesses, pneumonia, hospitalization and deaths. Because other pathogens can cause these outcomes, estimates of vaccine effectiveness underestimate the true protection afforded by vaccination. Nonetheless, numerous outbreaks of influenza have occurred in well-immunized nursing home populations. Moreover, a study in the US found that $58 \%$ of patients admitted to hospital over the age of $65 \mathrm{yrs}$, who were culture-positive for influenza, had received influenza vaccination in the preceding few months [19]. Accordingly, attempts are being made to augment protection through the use of adjuvants such as MF59, virosomal delivery of antigen to mucosaassociated lymphoid tissue, and the coadministration of live and inactivated vaccines.

\section{Safety of inactivated vaccines}

Many millions of doses of split product and purified surface antigen vaccines are administered throughout the world each year and the overall rate of adverse reactions is low. Postimmunization local erythema and tenderness are well documented, but the incidence of systemic symptoms and fever is similar following placebo or vaccine [20,21]. Severe cutaneous reactions (bullous pemphigoid and systemic vasculitis) have been described rarely, but their relation to vaccination is uncertain [22, 23]. Allergic reactions including anaphylaxis rarely occur following vaccination. Concern over vaccine safety among asthmatics and those with chronic airways disease has been raised because of a small number of adverse case reports [24]. Observational studies have found conflicting results with some postimmunization decreases in mean peak expiratory, flow increases in bronchial hyperreactivity and bronchodilator administration [25]. Other studies, including a large placebo-controlled, double-blinded crossover study have not found any exacerbations of asthma, change in peak flow or increased medication uses $[26-30]$. A recent review of the evidence 


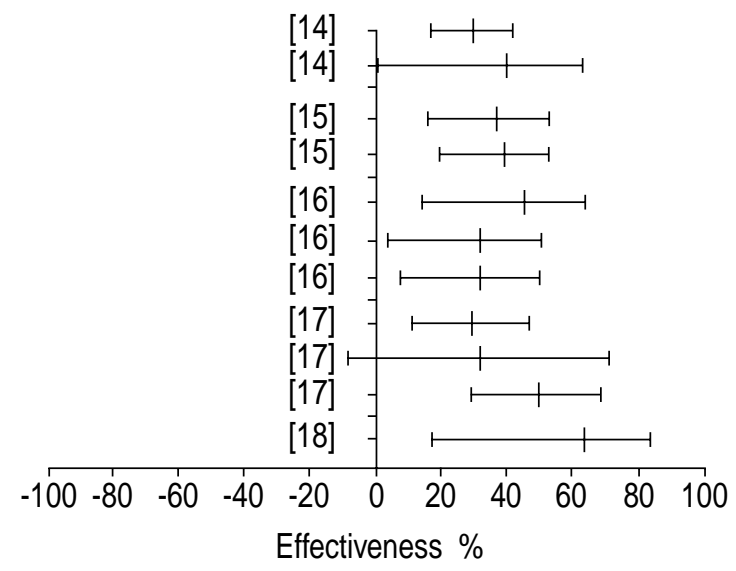

Fig. 2. - Influenza vaccine effectiveness in preventing pneumonia and influenza hospitalizations in the elderly. Data are presented as point estimates with $95 \%$ confidence intervals.

concluded that pulmonary function abnormalities may occur as a result of influenza vaccination, but the risks are very small and outweighed by the benefits of vaccination [31]. Guillain-Barré syndrome (GBS) was temporally associated with the 1976-1977 porcine influenza vaccination programme and the $1992-1993$ and 1993-1994 seasons. An association between GBS and other vaccine programmes has not been established. Analysis revealed an attributable rate of vaccine-related GBS of one case per 100,000 vaccinations with A/NewJersey/76 vaccine. During $1992-1993$ and $1993-1994$, the overall relative risk of 1.7 (95\% confidence intervals (CI) $1-2.8)$ for GBS was observed during the 6 weeks postvaccination [28]. This equates to one or two cases per million vaccinees. Persons with a history of GBS are at a higher risk of subsequently developing GBS than persons without such a history. Thus it seems prudent to avoid influenza vaccination in persons with a history of GBS, particularly those who are not at high risk for severe influenza complications or who developed GBS within six weeks of previous influenza vaccination [32].

\section{Live cold-adapted reassortant vaccines}

The highest annual attack rates for influenza occur in children and observations suggest that schoolchildren are central to the spread of influenza within the community [33, 34]. A 3-yr study of $3-5$ yr old children found suboptimal antibody responses to inactivated influenza vaccine and little or no protection against infection [35]. A promising approach to the control of influenza is the use of live, cold-adapted (CA) reassortant vaccines. Attenuated CA strains suitable for use in children, are generated by genetic reassortment between a contemporary wild-type virus (which express current HA and NA antigens) and a $\mathrm{CA}$ donor of attenuation. Donors of attenuation are influenza A/Ann Arbor/6/60 (H2N2) and B/Ann Arbor/1/66. These donor strains have been extensively passaged at $25^{\circ} \mathrm{C}$ and $33^{\circ} \mathrm{C}$ (i.e. are cold-adapted), are temperature-sensitive (i.e. are unable to replicate at core body temperature), and attenuated. These properties are associated with polygenic mutations. $\mathrm{CA}$ reassortants have been evaluated in more than 10,000 subjects. They display high levels of phenotypic and genotypic stability and appear safe when administered to infants and children including those with asthma and cystic fibrosis. CA reassortants are not transmissible to close seronegative contacts, despite viral shedding for periods of up to 12 days [36-38].

Live attenuated vaccines are well tolerated, with minor upper respiratory symptoms including rhinorrhoea or sore throat developing in $5-10 \%$ of adults and children, and $\sim 5 \%$ of healthy young children experience fever $>37.6^{\circ} \mathrm{C}$ following live $\mathrm{CA}$ vaccination [39-42]. Excess systemic complaints including myalgia, headache and lethargy occur in $2-5 \%$ of healthy, mostly adult subjects, but fever is unusual [43]. Pulmonary function measurements and airway reactivity to methacholine remain unchanged in young healthy adults infected with $\mathrm{CA}$ reassortant vaccine $[42,44]$. CA vaccines are well tolerated by elderly nursing home residents and patients with asthma, chronic obstructive airways disease or cardiac conditions $[45,46]$.

By mimicking infection with the wild-type virus, the CA vaccine delivers a larger dose of antigen to bronchial lymphoid tissue than the inactivated vaccine and offers the advantage of a broader immunological response. This includes higher or equal serum antibody (immunoglobulin (Ig)G, IgA, IgM) to both the $\mathrm{HA}$ and NA, greater neutralizing antibody and local mucosal secretory $\operatorname{IgA}$ responses than parenteral vaccine $[37,39,47-50]$, and a reduction of the amount and frequency of viral shedding in comparison with the inactivated vaccine following infection [44].

The protective efficacy of $\mathrm{CA}$ reassortant vaccines against artificial challenge or naturally-occurring infection has been demonstrated in a large number of studies [36, 37, 39-41, 45-51]. In general, the level of protection has been comparable to that observed with inactivated vaccines. In a 5-yr study of 5,210 healthy children and adults aged $1-65$ yrs, bivalent CA vaccine was no better than inactivated vaccine in preventing culture-positive influenza or a four-fold rise in antibody titres during the influenza season [43]. The efficacy of the CA vaccine against directed strains may be similar to the efficacy at preventing influenza that is antigenically matched to the strain in the vaccine [52]. The efficacy of the $\mathrm{CA}$ vaccine in protecting against a significantly drifted influenza A (H3N2) epidemic strain was demonstrated recently in a 2-yr multicentre, double-blind, placebo-controlled trial [39]. During the first year of the study, 1,314 healthy children aged $15-71$ months received a 2-dose regimen of trivalent $\mathrm{CA}$ reassortment vaccine. An additional 285 children received one dose of vaccine or placebo. Systemic haemagluttination inhibition (HAI) antibody responses developed in $92 \%$ and $88 \%$ of seronegative children after the first dose of H3N2 and influenza B vaccines, respectively and 96\% had antibodies after the second dose. However, only $16 \%$ developed antibodies to the first dose of H1N1 vaccine and $61 \%$ after the second. In year 1 , among those receiving a single dose of the $\mathrm{CA}$ vaccine, the efficacy of the vaccine in preventing culture-confirmed 
influenza was $87 \%(95 \%$ CI $47-97)$ for influenza A (H3N2) and 91\% (95\% CI 45-99) for influenza B. The efficacy after two doses was $96 \%(95 \%$ CI $90-99)$ for influenza A (H3N2) and 91\% (95\%CI 78-96) for influenza B. Vaccinees had 30\% fewer episodes of febrile otitis media. In year 2, 1,358 children aged $26-85$ months, returned for revaccination. Unlike the first year, when the vaccine strains closely antigenically matched the circulating viruses (A/Wuhan/359/ 95 and $\mathrm{B} /$ Harbin) the outbreak during year 2 consisted largely of a variant, A/Sydney, not contained in the vaccine. Nonetheless, the vaccine was $86 \%$ efficacious, and illness in vaccine failures was milder with fewer days of fever than in placebo-recipients [52]. It has been suggested that mass vaccination of $70 \%$ of children with the CA influenza vaccine could provide substantial protection to the community at large [53].

\section{Other vaccines and vaccine developments}

The serum HAI antibody response to split and subunit influenza vaccines is generally lower in older people. This decrease in the immune response can be explained partly by variations of prevaccination HAI titre, chronic ill health and possibly nutritional status. Some individuals may respond poorly because of an age-related decline in immune function.

One approach to boost immunity in the elderly has been to co-administer intranasal CA reassortant live vaccine and conventional vaccine given by injection. The efficacy of this approach was evaluated over three years by a double-blind, placebo-controlled study in which 523 residents of nursing homes all received trivalent inactivated vaccine parenterally and were then randomized to receive either $\mathrm{H} 3 \mathrm{~N} 2 \mathrm{CA}$ vaccine or placebo [54]. The overall occurrence of influenza A was low. Laboratory-confirmed influenza outbreaks occurred in three homes over 3 yrs. In these homes, subjects who received the CA vaccine had significantly lower rates of laboratory-documented influenza (9/162 versus 24/169; 61\% efficacy, 95\% CI 18-82) [54]. Following simultaneous $\mathrm{CA}$ and inactivated vaccination in seronegative young volunteers, serum HAI responses reached a greater magnitude as opposed to the inactivated vaccine only, and were sustained over 3 months [47].

Various adjuvants have been used with influenza vaccines in an attempt to enhance immunogenicity, but this has typically been associated with increased reactogenicity. Influenza vaccine containing MF-59, an oil in water emulsion with squalene as the oil component and sorbitan trioleate (an oil soluble surfactant), was licensed in Italy in 1997 [55]. Comparative studies involving 4,100 subjects who were randomized to receive MF-59 adjuvanted trivalent vaccine or commercial comparator vaccines reveal significant increases of $5-8 \%$ of subjects responding with fourfold increases in HAI antibody to $\mathrm{H} 3 \mathrm{~N} 2, \mathrm{H} 1 \mathrm{~N} 1$ and influenza B antigens adjuvanted with MF-59, in comparison with conventional vaccines, and the postvaccination geometric mean titres were $13-31 \%$ higher (A. Podda, Chiron Vaccines, personal communication). MF-59 has a dramatic effect on the antibody responses of immunologically-naïve subjects. A small comparative study of two doses $(7.5 \mu \mathrm{g}-30 \mu \mathrm{g})$ given on days 0 and 21 of conventional subunit $\mathrm{H} 5 \mathrm{~N} 3$ vaccine and vaccine adjuvanted with MF-59 found that the numbers who attained HAI titres of $\geqslant 1: 40$ or neutralizing antibody titres $\geqslant 1: 20$ were significantly higher on day 42 after the adjuvanted vaccine. (HAI 42\% versus 3\%: neutralizing antibody 94\% versus $22 \%$ ) [52].

Liposomes are lipid membrane particles that can serve as delivery systems for vaccine antigens and immunostimulators. Another type of liposome referred to as a virosome is created by inserting virus fusion proteins (the HA for influenza) into a liposome bilayer. A virosomal influenza vaccine has been licensed in Switzerland. Two small randomized trials have compared the immunogenicity of virosomal influenza vaccine with that of whole and subunit vaccines in the elderly. Improved antibody responses were noted with the virosomal formulation, which for some comparisons reached statistical significance $[57,58]$.

Because of the large number of embryonated hens' eggs required for the production of current commercially-available vaccines, advanced planning must start nearly a year before vaccination. The interval between identification of a new pandemic strain and outbreaks may be insufficient to produce vaccine using current technology. Moreover, in the event of a pandemic, vaccine production is unlikely to match global needs. For these and other reasons, the WHO has recognized an urgent need to develop cell culture techniques for influenza vaccine production. Vaccines prepared using Madin-Darby-Canine-Kidney (MDCK) and Vero cells (derived from human embryonic lung fibroblasts) have been given limited clinical trials. These cell culture systems offer the advantage of increasing vaccine production at short notice to meet unexpected demand or supplemental vaccine can be produced if a new antigenic variant is detected.

An additional alternative to egg-derived antigen involves the use of haemagglutinins expressed in insect cells by recombinant baculoviruses. Potential difficulties with these vaccines include the use of uncleaved rather than cleaved haemagglutinin and differences in glycosylation in insect as opposed to mammalian cells. None the less, the responses of adults to doses of $15-45 \mu \mathrm{g}$ of recombinant $\mathrm{H} 1$ and $\mathrm{H} 3$ antigens is similar to the responses to licensed vaccines. There is a dose-related response with serum HAI and neutralizing antibody titres equal or significantly higher using $90-135 \mu \mathrm{g}$ haemagglutinin than with subunit inactivated vaccine containing $15 \mu \mathrm{g}$ of egg-derived HA [59-61]. Another recent approach has been the development of nucleic acid vaccines. Deoxyribonucleic acid (DNA) sequences encoding the gene for the antigenic protein of interest can be integrated into bacterial plasmids, grown, purified and then inoculated into the host. After being injected, the plasmid enters a host cell, remaining in the nucleus as an episome. Expression of the plasmid DNA produces the antigenic protein that, in turn, hopefully produces 
an immune response. A protein produced by a plasmid-transfected cell is more likely to be folded in its natural configuration favouring neutralizing antibody formation and is more likely to be displayed on the surface by human leukocyte antigen (HLA) class I molecules inducing cytotoxic $\mathrm{T}$ cell responses [61, 63]. Experiments in mice have shown that influenza HAspecific IgG and IgA can be induced by both intramuscular and intranasal administrations of plasmid containing HA sequences, and further development is awaited with interest [64].

One of the problems associated with the use of current inactivated vaccines is the need to give repeated annual injections. The mucosal delivery of influenza vaccine would avoid the use of syringes and improve compliance. Relatively few antigens evoke humoral and secretory antibody responses when in contact with mucosal surfaces. Cholera toxin (CT) and Escherichia coli heat labile enterotoxin (LT) are potent mucosal immunogens and adjuvants in animal models. As a consequence of these properties, nontoxic LT mutants that retain adjuvant activity are being developed and clinical trials of such an adjuvanted vaccine are likely to proceed in the near future. Workers in Switzerland have recently evaluated a trivalent intranasal virosomal influenza vaccine with and without $E$. coli heat labile enterotoxin. Two spray inoculations given with an interval of 1 week induced a humoral response which was comparable to that with a single parenteral vaccination of virosomal vaccine containing the same total of influenza HA content [65]. In addition, a significantly higher induction of virus-specific $\operatorname{IgA}$ was noted in the saliva after two intranasal applications.

\section{Vaccine recommendations}

National authorities of many countries in Europe and North America recommend influenza vaccination for those $>65$ yrs of age; residents of nursing homes and other chronic care facilities that house persons of any age who have chronic medical conditions; adults and children with chronic cardiopulmonary conditions including asthma, and those with chronic medical conditions including metabolic disease and diabetes, renal dysfunction and immunosuppression [66]. Despite these guidelines, vaccine uptake rates remain variable and generally poor with $<20 \%$ asthmatics vaccinated in the UK [67]. There may also be medical, social and economic benefits of preventing influenza in young children and the working population especially healthcare workers [68-70].

\section{Antiviral therapy}

\section{M2 ion channel inhibitors}

The adamantanes, amantadine and rimantadine were discovered in the 1960 s to inhibit strains of influenza A. They are C-10 tricyclic primary amines that inhibit viral uncoating by their effects on the M2 ion channel. They inhibit human $\mathrm{H} 1 \mathrm{~N} 1, \mathrm{H} 2 \mathrm{~N} 2$, and
H3N2 subtypes of influenza A. Nonhuman subtypes of influenza A are also sensitive, indicating that future human pandemic strains will also be susceptible. At therapeutic doses, they have no effect on influenza B or other respiratory viral pathogens [67]. Amantadine is well absorbed with concentrations in nasal mucus approximately half those in plasma. Amantadine is excreted renally and can cause minor central nervous system (CNS) adverse effects such as insomnia, dizziness and lowered seizure threshold, particularly in the elderly and those with impaired renal function [72]. It is contraindicated in severe renal impairment and patients with epilepsy, and should be used in caution in elderly patients in whom renal function is likely to be impaired, i.e. many of those in the higher risk groups for influenza. Amantadine is extensively metabolized by the liver $(\sim 65 \%)$ and kidney $(20 \%)$ and is also excreted as unchanged drug by the kidney. Rimantadine concentrations in nasal mucus are $\sim 1.5$ times greater than in plasma. Comparative studies indicate that rimantadine is better tolerated than amantadine at equivalent doses.

Both drugs are effective for the treatment of acute influenza A infection, if commenced within $24 \mathrm{~h}$ of illness onset, reducing fever and symptoms by $1-2$ days and allowing earlier return to work [71, 73-75]. Chemoprophylaxis taken daily during influenza season reduces infection rates by $50-90 \%$ over the outbreak period [72, 76-78]. Administration of a drug as prophylaxis and treatment to nursing home residents during outbreaks of influenza within the facility may interrupt transmission but has led to the emergence and transmission of drug-resistant strains. Postexposure prophylaxis of household contacts prevents influenza A illness by $\sim 70 \%$, but drug resistance may emerge rapidly and render prophylaxis ineffective if the index case is also treated simultaneously [79]. The genetic basis for resistance appears to be single amino acid substitutions at positions 26, $27,30,31$ or 34 in the transmembrane portion of the M2 ion channel [80]. Amantadine-resistant viruses are resistant to rimantadine and vice-versa.

\section{Neuraminidase inhibitors}

The enzyme neuraminidase (NA) (sialidase) is a tetramer composed of a cytoplasmic tail, transmembrane domain, stalk region and globular head. Sialic acid is an integral part of the influenza virus receptor. The active site of NA lies within the head and is a highly conserved region among influenza $\mathrm{A}$ and $B$ viruses [81]. Because of its essential role in viral replication, NA represents a potential target for antiviral therapy. By analysing its crystallographic structure and interaction with sialic acid, selective reverse competitive inhibitors have been developed [82]. There are two licensed drugs: zanamivir and oseltamivir and a third, RWJ-270201 is in Phase II clinical studies. All three compounds are highly active against a broad range of influenza $A$ of human and avian origin and $\mathrm{B}$ viruses, including all nine NA subtypes in the avian reservoir. They tend to be less active in vitro against influenza B than influenza A 
neuraminidase. All three compounds inhibit amantadine and rimantadine resistant strains of influenza A.

Zanamivir has poor oral bioavailability and consequently has been administered topically. The licensed formulation is administered as a dry-powder aerosol. This may pose practical difficulties in the very young, frail or confused patients, although $74 \%$ of nursing home residents in one study had no difficulty with compliance [83]. In healthy volunteers, a mean of $13 \%$ (range $4-21 \%$ ) of the $10 \mathrm{mg}$ dose from the Diskhaler ${ }^{\mathrm{TM}}$ device is deposited in the bronchi and lungs and $78 \%$ is deposited in the oropharnynx [84]. Deposition studies have not been conducted in subjects with influenza or patients with obstructive airways disease. Nonetheless, the data from the lung deposition studies suggest that the concentration of zanamivir throughout the airways is far in excess of the median inhibitory concentration (IC50) values for zanamivir against the viral neuraminidases. Zanamivir remains detectable in sputum for as long as $24 \mathrm{~h}$ after dosing [85]. After oral administration, a median of $10-20 \%$ of the dose is systemically absorbed. Approximately $90 \%$ of intravenously administered zanamivir is excreted unchanged in the urine. Given the wide safety margin of zanamivir the possible increased exposure to zanamivir in patients with renal failure does not indicate a reduction in dosage during administration by oral inhalation. Oseltamivir ethyl ester prodrug is well absorbed with bioavailability in excess of $80 \%$ and metabolized to the active oseltamivir carboxylate. Animal studies suggest good distribution throughout the respiratory tract.

\section{Clinical efficacy}

Both zanamivir and oseltamivir have been found to be effective in prevention and treatment of experimental influenza A infection in healthy volunteers [86-88]. Intravenous zanamivir was found to significantly reduce the cytokine and chemokine proinflammatory response seen in influenza infection [89].

\section{Acute treatment in naturally acquired influenza}

Treatment studies are summarized in table 1 and discussed here.

Zanamivir. A double-blind, placebo controlled study of aerosolized zanamivir, with or without intranasal delivery, was conducted during the 1994-1995 season in North America and Europe [90]. Four-hundred and seventeen adults presenting within $48 \mathrm{~h}$ of onset of influenza-like symptoms were enrolled, with 262 (63\%) having confirmed influenza infection. Overall, there was a reduction of 1 day $(20 \%)$ in the median duration of time to alleviation of symptoms. Among those infected patients who were febrile at enrolment $\left(>38^{\circ} \mathrm{C}\right)$ and began treatment within $30 \mathrm{~h}$ of onset of symptoms, there was a $40 \%$ reduction in the median time to alleviation of symptoms from 4-7 days. Patients who were afebrile at time of enrolment and those who began treatment beyond $30 \mathrm{~h}$ after onset of symptoms had no significant benefit. The antiviral spectrum and clinical effectiveness of zanamivir included both influenza A and B virus infections. The Southern Hemisphere group recruited 455 adults into a double-blind, placebo-controlled trial presenting within $36 \mathrm{~h}$ of onset of influenza-like symptoms in Australia, New Zealand and South Africa [91]. Seventy-one per cent of the patients had laboratory confirmed influenza infection $(67 \%$ influenza $\mathrm{A}$ and $33 \%$ influenza B). Self-administered inhaled zanamivir was used for a period of 5 days following enrolment. There was a mean reduction of 1.5 days in the median time to alleviation of symptoms for influenza-positive patients and a reduction of 2 days for those febrile $\left(>37.8^{\circ} \mathrm{C}\right)$ at the time of treatment. For afebrile patients, no benefit was observed and there was no difference between influenza A and B virus infections.

Table 1.-Summary of trials in treatment of naturally acquired or experimentally induced influenza infection

\begin{tabular}{|c|c|c|c|c|c|}
\hline Reference & Therapy and duration & $\begin{array}{l}\text { Patients (proved } \\
\text { influenza) }\end{array}$ & Age yrs & $\begin{array}{l}\text { Time to } \\
\text { treatment* }\end{array}$ & $\begin{array}{l}\text { Reduction in days to relief } \\
\text { of symptoms }\end{array}$ \\
\hline$[90]$ & $\begin{array}{l}\text { Zanamivir inhaled } \\
10 \mathrm{mg} \text { b.i.d. for } 5 \text { days }\end{array}$ & $417(63 \%)$ & $>13$ & $<48 \mathrm{~h}$ & 1 day $\left(2\right.$ days if $<30 \mathrm{~h}$ and $\left.>38^{\circ} \mathrm{C}\right)$ \\
\hline [91] & $\begin{array}{l}\text { Zanamivir inhaled } \\
10 \mathrm{mg} \text { b.i.d for } 5 \text { days }\end{array}$ & $\begin{array}{c}455(71 \%) \\
76 \text { 'high risk' }\end{array}$ & $>12$ & $<36 \mathrm{~h}$ & $\begin{array}{l}1.5 \text { days (high risk group } 2.5 \text { days } \\
\text { and reduced complication } \\
\text { and antibiotic use) }\end{array}$ \\
\hline [92] & $\begin{array}{l}\text { Zanamivir inhaled } \\
10 \mathrm{mg} \text { b.i.d for } 5 \text { days }\end{array}$ & $\begin{array}{c}777(66 \%) \\
109 \text { 'high risk' }\end{array}$ & $\geqslant 12$ & $\begin{array}{l}<48 \mathrm{~h} \\
<36 \mathrm{~h}\end{array}$ & $\begin{array}{l}1 \text { day } \\
1.5 \text { days }\end{array}$ \\
\hline [93] & $\begin{array}{l}\text { Zanamivir inhaled } \\
10 \mathrm{mg} \text { b.i.d for } 5 \text { days }\end{array}$ & $356(78 \%)$ & $>12$ & $<48 \mathrm{~h}$ & $\begin{array}{l}2.5 \text { days (reduced antibiotic use } \\
\text { and complication rates) }\end{array}$ \\
\hline [94] & $\begin{array}{l}\text { Oseltamivir } 75 \text { or } \\
150 \mathrm{mg} \text { b.i.d for } 5 \text { days }\end{array}$ & $629(60 \%)$ & $18-65$ & $\begin{array}{l}<36 \mathrm{~h} \\
\text { and } 38^{\circ} \mathrm{C}\end{array}$ & $\begin{array}{l}1.4 \text { days. (returned to normal } \\
\text { activity } 2-3 \text { days earlier; } \\
\text { reduction in sinusitis by } 50 \% \text { ) }\end{array}$ \\
\hline [95] & $\begin{array}{l}\text { Oseltamivir } 75 \text { or } \\
150 \mathrm{mg} \text { b.i.d for } 5 \text { days }\end{array}$ & $726(66 \%)$ & $18-65$ & $\begin{array}{l}<36 \mathrm{~h} \\
<24 \mathrm{~h}\end{array}$ & $\begin{array}{l}1.2-1.5 \text { days } \\
1.9 \text { days (Oseltamivir: better symptom } \\
\text { scores and less viral shedding) }\end{array}$ \\
\hline
\end{tabular}

\footnotetext{
*: Time after onset of symptoms to receipt of drug.
} 
A small subgroup of high risk patients $(n=76)$, primarily those with asthma, $>65$ yrs of age or with underlying metabolic or cardiac diseases, was included. This group had a longer duration of illness than the general population of the study with median time to alleviation of symptoms of 8 days as compared with 6 days in the placebo "low risk" population. There was a median reduction of 2.5 days to alleviation of symptoms in the high risk subgroup when treated with zanamivir. There was a $33 \%$ reduction $(p=0.004)$ in complications such as bronchitis and sinusitis and 25\% reduction $(\mathrm{p}=0.025)$ in antibiotic use in those high risk patients receiving zanamivir [91]. A further doubleblinded, placebo-controlled study of zanamivir recruited a total of 777 patients including 109 high risk patients within $48 \mathrm{~h}$ of onset of influenza-like symptoms [92]. Sixty-six per cent of patients enrolled had laboratory confirmed influenza. Zanamivir showed a clinically and statistically significant benefit with a 1 -day reduction in the time to alleviation of symptoms in the healthy group and a 1.5-day reduction in the high risk group if administered within $36 \mathrm{~h}$ of onset of symptoms. Overall, there was a $31 \%$ reduction $(p=0.049)$ in the complications of influenza and faster alleviation of fever [92]. Another placebo controlled trial of 356 patients ( $78 \%$ confirmed influenza infection) found that inhaled zanamivir received within $48 \mathrm{~h}$ of onset of symptoms provided a 2.5 -day reduction in time to alleviation of symptoms from $7.5-5$ days [93]. Pooled analysis of studies totalling 2,235 patients treated with inhaled zanamivir found an overall $5 \%$ reduction from 18 to $13 \%(\mathrm{p}=0.006)$ of antibiotic prescriptions in influenza-positive patients [96].

Oseltamivir. Similar benefits have been seen with oseltamivir when used within $36 \mathrm{~h}$ of onset of symptoms. A double-blind, placebo-controlled trial over the 1998 season recruited 629 healthy adults presenting with influenza-like symptoms of which 374 $(60 \%)$ were proved influenza-positive [94]. These patients were randomized to placebo or $75 \mathrm{mg}$ b.i.d. or $150 \mathrm{mg}$ b.i.d. of oseltamivir. For those with fever $>38^{\circ} \mathrm{C}$ and presenting within $36 \mathrm{~h}$ of onset of symptoms, the median duration of time to alleviation of symptoms was 1.5 days (30\% reduction), severity of illness as judged by patient score cards was reduced by $38 \%$ and there was earlier return to normal activities by $2-3$ days. There was a reduction in incidence of bronchitis and sinusitis from 15 to $7 \% \quad(p=0.03)$ complicating influenza infected patients in those receiving oseltamivir. No dose difference between the oseltamivir treatment arms was noted [94]. A larger double-blind, placebo-controlled study of 719 adult patients in Canada, Europe and China tested the efficacy of oseltamivir 75 or $150 \mathrm{mg} \mathrm{b.i.d}$. for 5 days within $36 \mathrm{~h}$ of onset of symptoms [95]. Four hundred and seventy-five patients $(66 \%)$ were confirmed to have influenza infection. The duration of illness was reduced by $29 \mathrm{~h}(25 \% \mathrm{p}=0.02)$ and $35 \mathrm{~h}(30 \% \mathrm{p}=0.01)$ with 75 and $150 \mathrm{mg}$ b.i.d., respectively. If treatment was initiated within $24 \mathrm{~h}$ of onset of symptoms, duration of illness was shortened by $43 \mathrm{~h}(37 \% \mathrm{p}=0.02)$ and $47 \mathrm{~h}(40 \% \mathrm{p}=0.01)$ with 75 and $150 \mathrm{mg}$, respectively, compared with placebo. Patients who received oseltamivir had significantly lower viral titres in nose and throat swabs without impairing humoral (haemagglutinin inhibition) immune response to infection [95].

\section{Prevention}

A summary of seasonal and postcontact prophylaxis studies is shown in table 2. A 4-week study of 1,107 subjects over the 1997 (H3N2) influenza season concluded that $10 \mathrm{mg}$ inhaled zanamivir once per day was $84 \%$ efficacious $(95 \%$ CI $39 \%-83 \%)$ in preventing febrile laboratory-confirmed influenza infection [97]. No significant adverse effects were associated with drug prophylaxis.

A large study of 1,559 adults found that $75 \mathrm{mg}$ oseltamivir, given orally either once or twice daily over 6 weeks of the winter 1997 - 1998, revealed a $74 \%$ reduction in laboratory proven cases of influenza [98]. In the area where the influenza attack rate was highest, oseltamivir was $82 \%$ efficacious in preventing clinical influenza infection. Both once-daily and twice-daily administration of oseltamivir were effective against influenza infection. There was a higher rate of gastrointestinal side effects in those receiving oseltamivir, although the frequency of drug discontinuation was similar in both treatment and placebo groups [98]. In a study of 548 mainly prevaccinated frail elderly nursing home residents, once daily oseltamivir provided further protective efficacy of $92 \%$ against influenza infection and a reduction in

Table 2. - Summary of seasonal and post contact prophylaxis with neuraminidase inhibitors

\begin{tabular}{|c|c|c|c|c|c|}
\hline Reference & $\begin{array}{l}\text { Prophylaxis } \\
\text { treatment }\end{array}$ & Duration & $\begin{array}{l}\text { Subjects } \\
\mathrm{n}\end{array}$ & Age yrs & $\begin{array}{l}\text { Protective efficacy } \\
\text { and comments }\end{array}$ \\
\hline [97] & $\begin{array}{l}\text { Zanamivir } 10 \mathrm{mg} \\
\text { inhaled b.i.d }\end{array}$ & $\begin{array}{l}\text { 4-week seasonal } \\
\text { over outbreak }\end{array}$ & 1107 & $18-69$ & $\begin{array}{l}67 \% \text { against laboratory-confirmed } \\
\text { influenza infection, } 84 \% \\
\text { against clinical illness. }\end{array}$ \\
\hline [98] & $\begin{array}{l}\text { Oseltamivir } 75 \mathrm{mg} \text { once } \\
\text { daily or b.i.d }\end{array}$ & $\begin{array}{l}\text { 6/52 seasonal } \\
\text { study }\end{array}$ & 1559 & $18-65$ & $\begin{array}{l}74 \% \text { against laboratory-confirmed } \\
\text { influenza infection, no difference } \\
\text { between once daily and b.i.d., no } \\
\text { emergence of resistance. }\end{array}$ \\
\hline [99] & $\begin{array}{l}\text { Oseltamivir } 75 \mathrm{mg} \\
\text { once daily }\end{array}$ & $\begin{array}{l}6 / 52 \text { seasonal study } \\
\text { in nursing home }\end{array}$ & 548 & Mean of 82 & $\begin{array}{l}92 \% \text { in prevaccinated group }(80 \%) \\
\text { reduction in complications. }\end{array}$ \\
\hline [100] & $\begin{array}{l}\text { Oseltamivir } 75 \mathrm{mg} \\
\text { once daily }\end{array}$ & $\begin{array}{l}\text { Household } \\
\text { contacts } 5 / 7\end{array}$ & $\begin{array}{l}377 \\
\text { households }\end{array}$ & $\begin{array}{l}\text { Healthy } \\
\text { adults }\end{array}$ & $82 \%$ \\
\hline
\end{tabular}


complications [99]. A postcontact prevention study of once daily oseltamivir for 5 days in healthy family members of 377 households reduced the likelihood of contacts developing influenza virus infection by $82 \%$ [100].

\section{Use in special groups}

There are limited data of neuraminidase inhibitors in the elderly and patients with high risk conditions including underlying cardiorespiratory disease. A small number of high risk patients included in two treatment studies of zanamivir were shown to have significant symptom benefit and reductions in complications and prescribed antibiotic treatment [91, 92]. Seasonal oseltamivir prophylaxis in elderly nursing home residents did not cause adverse events and reduced risk of influenza by $92 \%$ [99]. Results from studies of NA inhibitors in the immunocompromised, infants, and the frail elderly are awaited. No animal tetratogenicity has been reported but neuraminidase inhibitors pass through the placenta and also into breast milk and their use is best avoided in pregnancy unless there is life-threathening infection for the mother.

\section{Resistance}

Influenza viruses with reduced susceptibility to NA inhibitors have been isolated following sequential tissue culture passage of the virus in the presence of drugs. Two mechanisms of resistance have been identified. Firstly, resistant viruses may involve multiple mutations clustered close to or around the binding site of the viral haemagglutinin with its sialic acid receptors, thereby reducing efficiency of virus binding and decreasing dependence on NA function. Resistance may also arise from single amino acid substitutions in the highly conserved active site of neuraminidase in the central cleft of the global head [101]. There are differences in cross-resistance, e.g. substitution at position 119 leads to zanamivir resistance but maintained susceptibility to oseltamivir and RWJ270201 [102]. NA mutant variants replicate in cell culture, but have reduced virulence in animal models [103]. Emergence of clinical resistance requires further study but appears rare. A study of over 600 samples collected from oseltamivir-treated patients found five resistant variants by genotypic and neuraminidase assays, but these patients did not have a poorer clinical outcome [104]. Attempts to select in vitro influenza B mutants resistant to NA inhibitors have failed. However a zanamivir resistant influenza $B$ virus was recovered from a bone-marrow transplant recipient following two weeks of treatment [105].

\section{Adverse effects}

Most clinical trials of zanamivir and oseltamivir were performed in healthy volunteers and the drugs have low acute toxicity. In zanamivir treatment and prevention studies, minor adverse events such as nausea, diarrhoea, headache and cough occurred in $<3 \%$ of recipients, and the frequency of such events was similar between placebo and drug arms [90-93, 97]. There have been a number of post marketing reports of transient acute bronchospasm and reductions in spirometric lung volumes in some patients [106]. However, more serious deteriorations in respiratory function have been reported in some patients with pre-existing respiratory disease [107].

Oseltamivir has been well tolerated with no serious adverse effects reported in clinical treatment or prophylaxis trials. It is associated with mild and transient gastrointestinal disturbance during the first two days of administration. In clinical trials, dropout rates were low and similar between treatment and placebo arms [95, 96, 98-100].

\section{Current status}

Neuraminidase inhibitors offer advances in treatment and chemoprophylaxis over M2 inhibitors due to their broader antiviral spectrum, proven efficacy with reduction of symptoms and complications, less potential for emergence of clinically resistant strains and for combating emerging influenza strains such as avian $\mathrm{H} 5$ and $\mathrm{H} 9$ for which vaccination is unavailable [108]. There may also be benefits in terms of delaying the emergence of drug resistance by decreasing unnecessary prescriptions [91, 94]. There is however, a need to assess direct comparative studies between the drugs available, and to consider the effect of combination treatment.

Several concerns have been raised regarding the prescription of NA inhibitors. Recommendations for their use in symptomatic patients of all age groups could theoretically overwhelm primary care physicians during outbreaks of influenza and other pathogens such as respiratory syncytial virus (RSV). Moreover, the lack of a simple, inexpensive, rapid, sensitive and specific "bedside" test for identifying influenza could result in considerable unnecessary and inappropriate use. In addition, unexpected adverse effects such as possible exacerbations of pre-existing respiratory disease are always a potential problem with new therapies as they become more widely used. Delivery issues and compliance with intranasal administration in the frail elderly and young children may be problematic. Although no clinical resistance appeared in trial settings, careful surveillance is required, particularly in populations in whom delivery and compliance are suboptimal.

From a healthcare provider's perspective, the principal issue is whether use of NA inhibitors, in comparison with other healthcare interventions, is financially worthwhile in all symptomatic patients, in specific groups (e.g. high risk groups), or none at all. A formal health-economic study is needed to address these concerns [109].

It remains to be seen if the neuraminidase inhibitors fulfil their clinical potential. It should not be forgotten that "prevention is better than cure" and that the appropriate use of vaccines remains as priority. 


\section{References}

1. Nicholson KG. Clinical features of influenza. Semin Respir Infect 1992; 7: 26-37.

2. Glezen WP. Serious morbidity and mortality associated with influenza epidemics. Epidemiol Review 1982; 4: $25-44$.

3. Glezen P, Decker M, Perrotta D. Survey of underlying conditions of persons hospitalised with acute respiratory disease during influenza epidemics in Houston 1978-81. Am Rev Respir Dis 1987; 136: 550-555.

4. Hay AJ. Functional properties of the virus ion channels. In: Nicholson KG, Webster RG, Hay AJ, eds. Textbook of Influenza. Oxford, Blackwell, 1998, pp. $74-79$.

5. Scholtisrek C, Hinshow VS, Olsen CW. Influenza in pigs and their role as the intermediate host. In: Nicholson KG, Webster RG, Hay AJ, eds. Textbook of Influenza. Oxford, Blackwell, 1998, pp. 137-144.

6. Meiklejohn G, Eickhoff TC, Graves P. Antigenic drift and efficacy of influenza virus vaccines. $J$ Infect Dis 1978; 138: 618-624.

7. Yuen KY, Chan PKS, Peiris M, et al. Clinical features and rapid diagnosis of human disease associated with avian influenza H5N1 virus. Lancet 1998; 351: 467 471.

8. Peiris $\mathrm{M}$, Yuen $\mathrm{KY}$, Leung $\mathrm{CW}$, et al. Human infection with influenza H9N2. Lancet 1999; 354: 916-917.

9. Potter CW, Oxford JS. Determinants of immunity to influenza infection in man. Br Med Bull 1979; 35: 6975.

10. Meiklejohn G. Viral respiratory disease at Lowry Air Force base in Denver 1952-82. J Infect Dis 1983; 148: $775-784$.

11. Edmondson KN, Graham SM, Warburton MF. A clinical trial of influenza vaccine in Canberra. Med J Aust 1970; 2: 6-13.

12. Govaert TME, Thijs CTMCN, Masurel N, et al. The efficacy of influenza vaccination in elderly individuals. A randomised double blind placebo-controlled trial. J Am Med Assoc 1994; 272: $1661-1665$.

13. Nicol KL. Efficacy/clinical effectiveness of inactivated influenza virus vaccines in adults. In: Nicholson $\mathrm{KG}$, Webster RG, Hay AJ, eds. Textbook of Influenza. Oxford, Blackwell, 1998, pp. 358-372.

14. Foster DA, Talsma A, Furumoto-Dawson A. Influenza vaccine effectiveness in preventing hospitalisation for pneumonia in the elderly. Am J Epidemiol 1992; 136: $296-307$.

15. Fedson DS, Wajda A, Nicol JP, et al. Clinical effectiveness of influenza vaccination in Manitoba. JAMA 1993; 270: 1956-1961.

16. Ohmit SE, Monto AS. Influenza vaccine effectiveness in preventing hospitalisation among the elderly during influenza type A and type B seasons. Int $J$ Epidemiol 1995; 24: $1240-1248$.

17. Nicol KL, Wuoronma J, von Sternberg T. Benefits of influenza vaccination for low-, intermediate-, and high risk senior citizens. Arch Intern Med 1998; 158: 1769 1776.

18. Fleming DM, Watson JM, Nicholson S, et al. Study of the effectiveness of influenza vaccination in elderly in the epidemic of 1989-1990 using a general practice database. Epidemiol Infect 1995; 115: 581-589.

19. Betts RF, O'Brien D, Menegus M, et al. A comparison of the protective benefit of influenza vaccine in reducing hospitalisation of patients infected with influenza A or B. Clin Infect Dis 1993; 17: 573.

20. Margolis KL, Nichol KL, Poland GA, et al. Frequency of adverse reactions to influenza vaccine in the elderly: randomised placebo controlled trial. $J \mathrm{Am}$ Med Assoc 1990; 264: 1139-1141.

21. Govaert ThME, Dinant GJ, Aretz K. Adverse effects to influenza vaccine in elderly people: randomised double-blind placebo controlled trial. BMJ 1993; 307 : 988-993.

22. Madder R, Narendran A, Lewtas J. Systemic vasculitis following influenza vaccination - three cases and literature review. J Rheum 1993; 20: 1429-1431.

23. Fournier B, Deschamps V, Bouscarat F. Bullous pemphigoid induced by vaccination. Br J Derm 1997; 135: $153-155$.

24. Daggett P. Influenza and asthma. Lancet 1992; 339: 367.

25. Bell TD, Chai H, Berlow B. Immunisation with killed influenza vaccine in children with chronic asthma. Chest 1978; 73: 140-145.

26. Ahmed AH, Nicholson KG, Hammersley VS. Influenza vaccination in patients with asthma: effect on peak expiratory flow, asthma symptoms and use of medication. Vaccine 1997; 15: $1008-1013$.

27. Kava T. Acute respiratory infection, influenza vaccination and airway reactivity on asthma. Eur J Respir Dis: 1987: Suppl. 150. 1-38.

28. Retailliau HF, Curtis AC, Storr G, et al. Illness after influenza vaccination reported through a nation-wide surveillance system 1976-1977. Am J Epidemiol 1980; 111: $270-278$.

29. Albazzaz MK, Harvey JE, Grilli EA. Subunit influenza vaccination in adults with asthma: effect on clinical state, airways, and antibody response. $B M J 1987$; 294: 1196-1197.

30. Nicholson KG, Nyugen-Van-Tam JS, Ahmed AH. Randomised placebo controlled crossover trial on effect of inactivated influenza vaccine on pulmonary function in asthma. Lancet 1998; 351: 326-332.

31. Stephenson I, Nicholson KG. Influenza vaccination and asthma: current studies and recommendations. BioDrugs 1999; 11: 223 - 228.

32. Lasky T, Terracciano GJ, Magder L. The GuillainBarré syndrome and the 1992-93 and 1993-94 influenza vaccines. $N$ Engl J Med 1998; 339: $1797-$ 1802.

33. Nguyen-Van-Tam. Epidemiology of influenza. In: Nicholson KG, Webster RG, Hay AJ, eds. Textbook of Influenza. Oxford, Blackwell, 1998, pp. 181-206.

34. Taber LH, Paredes A, Glezen WP. Infection with influenza A/Victoria virus in Houston families. $J \mathrm{Hyg}$ Camb 1981; 86: 303-313.

35. Couch RB, Keitel WA, Cate TR et al. Prevention of influenza virus infections by current inactivated influenza vaccines. In: Brown LE, Hampson AW, Webster RG, eds. Options for the control of influenza. III. Elsevier, Amsterdam, 1999.

36. Gruber W, Taber L, Glezen W. Live attenuated and inactivated influenza vaccine in schoolage children. Am J Dis Child 1990; 144: 595-600.

37. Wright PF, Okabe N, McKee KT. Cold adapted recombinant influenza $\mathrm{A}$ virus vaccines in seronegative young children. $J$ Infect Dis 1982; 146: $71-79$.

38. Cha T, Kao K, Zhao J. Genotypic stability of coldadapted influenza vaccine in an efficacy clinical trial. $J$ Clin Microbiol 2000; 38: 839-845. 
39. Belshe RB, Mendelman PM, Treanor JJ. The efficacy of live attenuated cold adapted trivalent intranasal influenza virus vaccine in children. $N$ Engl J Med 1998; 338: $1405-1412$.

40. Belshe RB, Van Voris LP, Bartram J. Live attenuated influenza A virus vaccine in children: results of a field trial. J Infect Dis 1984; 150: 834-840.

41. Belshe R. Clinical status of attenuated influenza vaccines. Program and abstracts from Second International Symposium on Influenza and other Respiratory viruses. Dec 1999, Grand Cayman, British West Indies.

42. Atmar RL, Bloom K, Keitel W, et al. Effect of live attenuated cold recombinant influenza virus vaccine on pulmonary function in healthy and asthmatic adults. Vaccine 1990; 8: 217-224.

43. Edwards KM, Dupont WD, Westrich MK, et al. A randomised controlled trial of cold-adapted and inactivated vaccines for the prevention of influenza A disease. J Inf Dis 1994; 169: 68-76.

44. Goings SA, Kulle TJ, Bascom R. Effects of nitrogen oxide exposure on susceptibility to influenza A virus infection in healthy adults. Am Rev Respir Dis 1989; 139: $1075-1081$.

45. Gorse GJ, Belshe RB, Munn N. Superiority of live attenuated compared with inactivated influenza $\mathrm{A}$ virus vaccines in older chronically ill adults. Chest 1991; 100: $977-984$.

46. Treanor JJ, Dumyati G, O'Brien D, et al. Evaluation of cold adapted reassortant influenza $B$ virus vaccines in elderly and chronically ill patients. J Infect Dis 1994; 169: 402-407.

47. Betts RF, Treanor JJ. Approaches to improved influenza vaccination. Vaccine 2000; 18: 1690-1695.

48. Clements ML, Betts RF, Murphy BR. Advantage of live attenuated cold adapted influenza $A$ virus over inactivated vaccine for A/Washington/80. Lancet 1984; 1: 705-709.

49. Treanor JJ, Kotloff K, Betts RF, et al. Evaluation of live cold adapted and inactivated influenza vaccines in prevention of virus infection and illness following challenge with wild-type influenza A (H1N1), A (H3N2) and B viruses. Vaccine 1999; 18: 899-906.

50. Belshe RB, Gruber WL, Mendelson PM, et al. Correlates of immune protection induced by live attenuated cold adapted trivalent intranasal influenza virus vaccine. $J$ Inf Dis 2000; 181: 1133-1137.

51. Gruber W, Belshe RB, King JC, et al. Evaluation of live attenuated influenza vaccine in children of $6-18$ months: Safety, immunogenicity and efficacy. J Inf Dis 1996; 173: $1313-1319$.

52. Belshe RB, Gruber WC, Mendelman PM, et al. The efficacy of live attenuated cold adapted trivalent intranasal influenza virus vaccine in children. $J$ Paediat 2000; 136: $168-175$.

53. Longini IM, Halloran ME, Nizam A, et al. Estimation of the efficacy of live attenuated influenza vaccine from a two-year multicentre vaccine trial: implications for influenza epidemic control. Vaccine 2000; 17: $1902-1909$

54. Treanor JJ, Mattison HR, Dumyati G. Protective efficacy of combined live intranasal and inactivated influenza A virus vaccines in the elderly. Ann Int Med 1992; 117: 625-633.

55. DeDonato S, Granoff D, Minutello M, et al. Safety and immunogenicity of MF-59 adjuvanted influenza vaccine in the elderly. Vaccine 1999; 17: 3094-3101.

56. Nicholson KG. Phase I tolerability, safety and immunogenicity study of $\mathrm{H} 5 \mathrm{~N} 3$ vaccine in healthy adults. Program and abstracts from Second International Symposium on Influenza and other Respiratory viruses. Dec 1999, Grand Cayman, British West Indies.

57. Conne P, Gauthey L, Vernet P. Immunogenicity of trivalent subunit versus virosome-formulated influenza vaccines in geriatric patients. Vaccine 1997; 15: $1675-$ 1679.

58. Gluck R, Mischler R, Finkel B. Immunogenicity of new virosome influenza vaccine in elderly people. Lancet 1994; 344: 160-163.

59. Powers DC, Smith GE, Anderson EL. Influenza A virus vaccines containing purified recombinant $\mathrm{H} 3$ haemagglutinin are well tolerated and induce protective immune responses in healthy adults. $J$ Inf Dis 1995; 171: 1595-1599.

60. Laky DL, Treanor JJ, Betts RF. Recombinant baculovirus influenza A heamagglutinin vaccines are well tolerated and immunogenic in healthy adults. $J$ Inf Dis 1996; 174: $838-841$.

61. Treanor JJ, Betts RF, Smith GE. Evaluation of recombinant haemagglutinin expressed in insect cells as an influenza vaccine in young and elderly adults. $J$ Inf Dis 1996; 173: 1467-1470.

62. Robinson HL. Nucleic acid vaccines: an overview. Vaccine 1997; 15: 785-787.

63. Seder RA, Gurunathan S. DNA vaccines - designer vaccines for the 21st century. $N$ Engl J Med 1999; 341: 277-278.

64. Ban EM, van Ginkel KW, Simecka JW. Mucosal immunisation with DNA encoding influenza haemagglutinin. Vaccine 1997; 15: 811-814.

65. Glück U, Gebbers JO, Glück R. Phase I evaluation of intranasal virosomal influenza vaccine with and without Escherichia coli heat-labile toxin in adult volunteers. J Virol 1999; 73: 7780 - 7786.

66. Nicholson KG, Snacken R, Palache AM. Influenza immunisation policies in Europe and the United States. Vaccine 1995; 13: 365-369.

67. Wiselka MJ, Kent J, Stern M, Nicholson KG. Influenza and asthma. Lancet 1992; 339: 367-368.

68. Nichol KL, Margolis KL, Wuorenma J, et al. The efficacy and cost effectiveness of vaccination against influenza in elderly persons living in the community. $N$ Engl J Med 1995; 333: 889-900.

69. Barnett ED. Influenza immunisation for children. $N$ Engl J Med 1998; 338: 1459-1461.

70. Wilde JA, McMillan JA, Serwint J. Effectiveness of influenza vaccine in health care professionals. A randomised trial. J Am Med Assoc 1999; 281: 908 -913.

71. Smorodintsev AA, Zlydnikov DM, Kiseleva AM, et al. Evaluation of amantadine in artificially induced A2 and B influenza. J Am Med Assoc 1970; 213: 1448 1454.

72. Bryson YJ, Monahan C, Pollack M, et al. A prospective double blind study of side effects associated with administration of amantadine for influenza A prophylaxis. J Inf Dis 1980; 141: 543-547.

73. Van Voris LP, Betts RF, Hayden FG, et al. Successful treatment of naturally occurring influenza A/USSR/77 H1N1. J Am Med Assoc 1981; 245: 1128-1131.

74. Wingfield WL, Pollack D, Grunert RR. Therapeutic efficacy of amantadine and rimantadine $\mathrm{HCl}$ in naturally occurring influenza A2 respiratory illness in man. N Engl J Med 1969; 281: 579-584. 
75. Nicholson KG, Wiselka MJ. Amantadine for influenza A. BMJ 1991; 302: 425-426.

76. Dolin R, Reichman RC, Madore HP, et al. A controlled trial of amantadine and rimantadine in prophylaxis of influenza A infection. $N$ Engl J Med 1982; 307: $580-584$.

77. Togo Y, Hornich RB, Dawkins AT. Double blind studies designed to assess prophylactic efficacy of amantadine hydrochloride against A2/Rockville/1/65 strain. J Am Med Assoc 1968; 203: 1095-1099.

78. Clover RD, Crawford SA, Abell TD, et al. Effectiveness of rimantadine prophylaxis of children within families. Am J Dis Child 1986; 140: 706-709.

79. Hayden FG, Belshe RB, Clover RD, et al. Emergence and apparent transmission of rimantadine-resistant influenza A in families. $N$ Engl J Med 1989; 321: $1696-1702$

80. Hay AJ, Wolstenholme AJ, Skehel JJ, Smith MH. Molecular basis of resistance of influenza A viruses to amantadine. J Antimicrob Chemo 1986; 18: Suppl. B, $19-29$.

81. Varghese JN, Colman PM. Three-dimensional structure of neuraminidase of influenza virus A/Tokoyo/3/ 67 at 2.2A resolution. J Mol Biol 1991; 221: 473-486.

82. Von Itzstein $\mathrm{I}, \mathrm{Wu} \mathrm{WY}$, Kok $\mathrm{S}$, et al. Rational design of potent sialidase-based inhibition of influenza virus replication. Nature 1993; 363: $418-423$.

83. Lee C, Loeb M, Philips A. Use of zanamivir to control an outbreak of influenza A. 38th ICAAC San Diego Sept 1999 abstract 283.

84. Cass LMR, Brown J, Pickford M. Pharmacoscintigraphic evaluation of lung deposition of inhaled zanamivir in healthy volunteers. Clin Pharmacokine 1999; 36: Suppl. 1, 21-31.

85. Peng AW. Sustained concentration in respiratory tract after $10 \mathrm{mg}$ zanamivir inhalation. 38th ICAAC San Diego Sept 1999 abstract 46.

86. Hayden FG, Treanor JJ, Betts RF, et al. Safety and efficacy of the neuraminidase inhibitor GC167 in experimental human influenza. JAMA 1996; 275: 295-299.

87. Hayden FG, Treanor JJ, Fritz RS, et al. Use of the oral neuraminidase inhibitor oseltamivir in experimental human influenza- randomised controlled trials for prevention and treatment. JAMA 1999; 282: $1240-1247$.

88. Calfee DP, Peng AW, Hussey EK. Protective efficacy of reduced frequency dosing of intranasal zanamivir in experimental human influenza. 38th ICAAC San Diego Sept 1999 abstract H68.

89. Fritz RS, Hayden FG, Calfee DP. Cytokine and chemokine responses during experimental influenza A infection and effect of intravenous zanamivir. 38th ICAAC San Diego Sept 1999 abstract H56.

90. Hayden FG, Osterhaus ADME, Treanor JJ, et al. Efficacy and safety of neuraminidase inhibitor zanamivir in the treatment of influenzavirus infections. $N$ Engl J Med 1997; 337: 874-880.

91. MIST Study Group. Randomised trial of efficacy and safety of inhaled zanamivir in treatment of influenza A and B virus infection. Lancet 1998; 352: 1877-1884.

92. Lalezari J, Klein T, Stapleton JT, et al. The efficacy and safety of inhaled zanamivir in the treatment of influenza in healthy and high risk individuals in North America. J Antimicrob Chemother 1999; 44: 42 abstract $\mathrm{P} 8$.

93. Fleming D, Makela M, Pauksens K, et al. High risk and otherwise healthy patients demonstrate alleviation of influenza symptoms 2.5days earlier following inhaled zanamivir treatment: European study, winter 1997/8. IDSA 1998 Denver; abstr 789.

94. Treanor JJ, Hayden FG, Vrooman PS, et al. Efficacy and safety of oral neuraminidase inhibitor oseltamivir in treating acute influenza. JAMA 2000; 283: $1016-$ 1025 .

95. Nicholson KG, Aoki FY, Osterhaus ADME, et al. Efficacy and safety of oseltamivir in treatment of acute influenza: a randomised controlled trial. Lancet 2000; 355: $1845-1850$.

96. Monto AS, Webster A, Keene O, et al. Randomised placebo-controlled studies of inhaled zanamivir in the treatment of influenza A and A: pooled efficacy analysis. J Antimicrob Chemother 1999; 44: 23-29.

97. Monto AS, Robinson DP, Hollocher ML, et al. Zanamivir in prevention of influenza among healthy adults - a randomised controlled trial. $\mathrm{J} \mathrm{Am} \mathrm{Med}$ Assoc 1999; 282: 31 - 38 .

98. Hayden FG, Atmar RL, Schilling M, et al. Use of selective oral neuraminidase inhibitor oseltamivir to prevent influenza. $N$ Engl J Med 1999; 341: $1336-$ 1345.

99. Peters P. Oseltamivir is effective in prophylaxis of influenza in vaccinated frail elderly. Second International Symposium on Influenza and other Respiratory viruses. Dec 1999, Grand Cayman, British West Indies.

100. Oxford J. Short term prophylaxis with oral oseltamivir effectively prevents spread of influenza A and B. Second International Symposium on Influenza and other Respiratory viruses. Dec 1999, Grand Cayman, British West Indies.

101. Staschke KA, Colacino JM, Baxter AJ. Molecular basis for the resistance of influenza viruses to 4guanidino-Neu5Ac2en. Virology 1995; 214: 642-646.

102. McKimm-Breschkin JL, Sahasrabudhe A, Blick TJ. Mutations in a conserved residue in the influenza virus neuraminidase active site decreases sensitivity to Neu5Ac2en-derived inhibitors. J Virol 1998; 72: $2456-2462$.

103. Ives J, Carr J, Roberts N. An oseltamivir-treatment selected influenza A/N2 virus with an R292K mutation in the neuraminidase gene has reduced infectivity in vivo. Second International Symposium on Influenza and other Respiratory viruses. Dec1999, Grand Cayman, British West Indies.

104. Roberts N. Update on resistance to neuraminidase inhibitors. Second International Symposium on Influenza and other Respiratory viruses. Dec 1999, Grand Cayman, British West Indies.

105. Gubareva LV, Matrosovich MN, Brenner MK, et al. Evidence for zanamivir resistance in an immunocompromised child infected with influenza B virus. $J$ Inf Dis 1998; 178: 1257-1262.

106. Gubareva LV, Kaiser L, Hayden KG. Influenza virus neuraminidase inhibitors. Lancet 2000; 355: 827-835.

107. Williamson JC, Pegram PS. Respiratory distress associated with zanamivir. $N$ Engl J Med 2000; 342: $661-662$.

108. Webster R. Efficacy of oseltamivir on H5N1 and H9N2 influenza viruses. Second International Symposium on Influenza and other Respiratory viruses. Dec 1999, Grand Cayman, British West Indies.

109. Wenzel RP. Expanding the Treatment options for influenza. J Am Med Assoc 2000; 283: 1057-1058. 